\title{
Generation of Basis Vectors for Magnetic Structures and Displacement Modes
}

\author{
Z. L. Davies and A. S. Wills \\ Department of Chemistry, University College London, 20 Gordon Street, London WC1H 0AJ, UK \\ Correspondence should be addressed to A. S. Wills; a.s.wills@ucl.ac.uk \\ Received 19 September 2016; Accepted 25 October 2016 \\ Academic Editor: Jörg Fink
}

Copyright (C) 2016 Z. L. Davies and A. S. Wills. This is an open access article distributed under the Creative Commons Attribution License, which permits unrestricted use, distribution, and reproduction in any medium, provided the original work is properly cited.

Increasing attention is being focused on the use of symmetry-adapted functions to describe magnetic structures, structural distortions, and incommensurate crystallography. Though the calculation of such functions is well developed, significant difficulties can arise such as the generation of too many or too few basis functions to minimally span the linear vector space. We present an elegant solution to these difficulties using the concept of basis sets and discuss previous work in this area using this concept. Further, we highlight the significance of unitary irreducible representations in this method and provide the first validation that the irreducible representations of the crystallographic space groups tabulated by Kovalev are unitary.

\section{Introduction}

The use of symmetry-adapted functions is well established in many fields, such as electronic structure calculations and vibrational mode analysis. Increasingly, its value to crystallography is being recognized too. Their direct application in the refinement of magnetic structures was originally developed in SARA $h$ [1] and is also available in Fullprof [2] and for commensurate systems in TOPAS [3]. More recent interest has focused upon developing the application of this technique to displacive phase transitions [4-9].

In magnetism, analysis using these functions has been applied to several neutron scattering techniques, including spherical neutron polarimetry [10], single crystal diffraction $[11,12]$, and powder diffraction [13-15].

The foundation of this method is the derivation of symmetry-adapted functions using the techniques of representation theory. In magnetism and crystallography these functions are usually referred to as basis vectors (BVs) and they define the order parameters of some property resulting from a phase transition; the coefficients of the BVs define the state of a system with respect to a reference. As such, via the Landau theory of a second order phase transition, they have physical meaning that in magnetism can also be related to the eigenfunctions of a spin Hamiltonian [1619]. The generality of the techniques means that different local quantities can be studied within the same mathematical framework, linking the physics and symmetry considerations of order-disorder transitions based on the ordering of scalar quantities, polar vectors (atomic displacements), axial vectors (atomic moments), and more complex local structures, such as tensor and quadrupolar order [20,21].

Constructing BVs is a simple, though laborious, task [22]. A representation of some system property under its symmetry operations is constructed as the direct product of two component representations $\Gamma=\Gamma_{V} \otimes \Gamma_{\text {Perm }}$. In this notation, $\Gamma_{\text {Perm }}$ represents the permutation of atoms under the symmetry operations of the system; $\Gamma_{V}$ represents how some property of interest transforms under the same operations. Fortunately, the full matrix representation is not required and the character of the matrices is sufficient. Once these have been determined, $\Gamma$ is reduced to a linear combination of irreducible representations (IRs): $\Gamma=\sum_{i} C^{i} \Gamma^{i}$. The final step is the generation of BVs using the method of projection operators.

A common problem when performing these calculations is the projection of too few or too many basis vectors. Undergeneration has been thoroughly explored by Stokes 
et al. [23]; however, the problem of overgeneration has not been previously resolved. In this work we discuss projection operators using the concept of "basis sets" and apply use concept to resolve over- and undergeneration; we illustrate the general procedure with an example. Further, in Section 6, we discuss the unitary nature of the IRs listed in the tables of Kovalev [24] and highlight the importance of this property. We begin by defining what constitutes an appropriate set of solutions and how they are derived.

\section{Properties of Basis Sets}

Any property of a crystal, such as its magnetic structure or atomic displacements, may be described by a field. The axis system of such a field is arbitrary, and the system and is divisible into subspaces, each with the symmetry of one IR in the linear expansion $\Gamma=\sum_{i} C^{i} \Gamma^{i}$. Each subspace is spanned by sets of BVs with appropriate symmetry, basis sets. The symmetric basis of the whole system is given by the set of all basis sets, the complete linear vector space. The desired basis for the subspace which transforms as $\Gamma^{v}$ is a set of vectors $\left\{\psi_{1}^{\nu}, \psi_{2}^{\nu}, \ldots, \psi_{d^{v}}^{\nu}\right\}$ for which

$$
g \psi_{l}^{\nu}=\sum_{m=1}^{d^{v}} \mathfrak{d}_{m l}^{\nu}(g) \psi_{m}^{\nu}, \quad \forall g \in \mathbb{G} .
$$

Here $\mathbb{G}$ is the symmetry group of the system, $\mathfrak{D}^{\nu}(g)$ is the matrix representing the operation $g$ in the IR $\Gamma^{\nu}$, and $d^{\nu}$ is the order of $\Gamma^{\nu}$. A set of BVs obeying (1) will be referred to as a basis set; note that basis sets always occur in sets of order $d^{v}$. We will use the properties of basis sets to explore over- and undergeneration in Sections 4 and 5.

\section{Presentation of the Operators}

Here we present the key equations in the method of projection operators in two forms [25] and discuss the importance of unitary IRs when using these standard forms. The reduction formula is defined as

$$
\frac{1}{|\mathbb{G}|} \sum_{g_{s} \in \mathbb{G}} \chi^{\nu}\left(g_{s}\right) \chi^{\mu}\left(g_{s}^{-1}\right)=\delta_{\nu, \mu}
$$

where $\chi^{n}\left(g_{s}\right)$ is the character of the matrix representing $g_{s}$ in the representation $\Gamma^{n}$. The action of the reduction operator is to determine the coefficients, $C^{\mu}$, when applied to some representation $\Gamma^{\mu}=\sum_{\mu} C^{\mu} \Gamma^{\mu}$. When a matrix is the conjugate transpose of its inverse, then it is said to be unitary; an IR composed of unitary matrices is, itself, unitary. Under the assumption of unitary IRs (2) can be rewritten in its usual form as follows:

$$
\begin{aligned}
\mathfrak{b}_{m l}\left(g_{s}\right) & =\mathfrak{d}_{l m}^{*}\left(g_{s}^{-1}\right), \\
\frac{1}{|\mathbb{G}|} \sum_{g_{s} \in \mathbb{G}} \chi^{v}\left(g_{s}\right) \chi^{\mu *}\left(g_{s}\right) & =\delta_{\mu, v} .
\end{aligned}
$$

Similarly there are two forms of the projection operator (strictly, the operators presented in (4) and (5) are not projection operators; this name is reserved for their idempotent derivatives for which $l=m$; however, the phrase "method of projection operators" is synonymous with technique and therefore we shall refer to them by this name from herein). The first of these is applicable to any IR, while the second line is the projection operator derived under the assumption of unitary IRs.

$$
\begin{aligned}
W_{m l}^{\mu} \psi_{i}^{\nu} & =\frac{d^{\mu}}{|\mathbb{G}|} \sum_{g_{s}} \mathfrak{b}_{m l}^{\mu}\left(g_{s}^{-1}\right) \mathfrak{I}\left(g_{s}\right) \psi_{i}^{\nu}, \\
W_{l m}^{\mu} & =\frac{d^{\mu}}{|\mathbb{G}|} \sum_{g_{s}} \mathfrak{b}_{l m}^{\mu *}\left(g_{s}\right) \mathfrak{I}\left(g_{s}\right), \\
W_{l m}^{\mu} \psi_{m}^{\mu} & =\psi_{l}^{\mu} .
\end{aligned}
$$

The projection operator $W_{l m}^{\mu}$ generates a set of vectors of order $d^{\mu}$ from a single vector by permuting the BVs amongst themselves; specifically a group of BVs the symmetry of $\Gamma^{\mu}$. Thus, the projection operator will "project out" a complete basis set from a single BV. However, in general we will not know any of the BVs and we must consider the action of the projection operator upon a trial vector, $\phi=\sum_{\nu} \sum_{i}^{d^{v}} C_{i}^{v} \psi_{i}^{v}$.

$$
W_{l m}^{\mu} \phi=\sum_{\nu} \sum_{i}^{d^{\nu}} C_{i}^{\nu} W_{m l}^{\mu} \psi_{i}^{\nu}=\sum_{\nu} \sum_{i}^{d^{\nu}} C_{i}^{\nu} \psi_{l}^{\mu} \delta_{m, i} \delta_{\mu, \nu}=C_{m}^{\mu} \psi_{l}^{\mu}
$$

The action of the projection operator is to take the component of $\phi$ along $\psi_{m}^{\mu}$ and transform it into $\psi_{l}^{\mu}$; all the other components are transformed to zero. The projection operator is most commonly encountered in the form of (5), which restricts the IRs to being unitary; we discuss this restriction in Section 6.

\section{Overgeneration}

Overgeneration is the projection of more BVs than required to minimally span system's degrees of freedom. Correspondingly, some of the derived BVs are linearly related. The problem is how best to reduce our set of solutions to one that is minimal while preserving the characteristic symmetry properties within our basis.

Often equivalent solutions will occur in pairs, related by a complex coefficient: $\psi_{1}=C \psi_{2}$. In this case it is simple for an algorithm to determine the equivalence relationships. However, when three or more BVs are linearly related then, because our BVs are not required to be orthogonal, there is no simple way to determine linear relationships between them. In particular, there is no simple algorithm to determine equivalence relations and thus calculate which BVs are equivalent. The most often used technique to resolve this problem is Gram Schmidt orthogonalisation, which involves generating a set of linearly-independent functions by successively projecting out components that are orthogonal to a previously determined set of vectors. Its difficulty is that it removes the natural symmetry of the BVs. It also fails to recognise that only entire basis sets can be eliminated; otherwise our solution is not a set of basis sets and cannot have the correct symmetry properties. 
Rephrasing the problem, as the projection operator must derive a set of order $d^{v}$ or 0 for each trial, it is apparent that when overgeneration occurs too many trial functions have been used. If, through judicious choice of trial vectors, we can reduce all BV equivalencies to the form $\psi_{1}=C \psi_{2}$, then it becomes possible to determine which trial vectors give equivalent answers and eliminate all but one of them. By eliminating trial vectors and not basis vectors, the symmetry properties of the BVs are preserved.

4.1. Symmetry-Adapted Trial Vectors. Our goal is to determine a method of constructing an appropriate set of trial functions. If two BVs are linearly related then the system property at each point must have the same linear relationship. Thus, by controlling how the property at a single point is generated we can control the generation of the entire BV. Under the method of projection operators, the property at a single point is generated by the sum action of all the operators which generate that point from an initial position $A_{0}$; if we consider $A_{0}$ itself then these operators are the so-called "stabilizers" of $A_{0}$, denoted $\mathbb{W}_{0}$.

$$
\begin{gathered}
\mathbb{H}_{0} \subset \mathbb{G}_{k}, \\
\frac{d^{\mu}}{|\mathbb{G}|} \sum_{h_{s} \in \mathbb{H}_{0}} \mathfrak{d}_{m l}^{\mu}\left(h_{s}^{-1}\right) \mathfrak{I}\left(g_{s}\right) \phi_{i}^{\nu}=\left(\begin{array}{c}
a_{0} \\
b_{0} \\
c_{0}
\end{array}\right) A_{0} .
\end{gathered}
$$

The property at atom $A_{0}$ is defined by the vector $\left(a_{0}\right.$, $\left.b_{0}, c_{0}\right)$, defined by the crystallographic axes. Izyumov and Syromyatnikov [22] have developed a formalism for the reduction and projection operators using stabilizers.

The stabilizers of $A_{0}$ form a group and thus subdivide the $R^{3}$ space into invariant subspaces; by selecting our trial vectors to lie within lines and planes of invariance we naturally simplify the relations between our projected BVs. In many cases it will be obvious where the lines and planes of invariance lie for a stabilizer group, when it is not we can construct them using the method of projection operators twice. First, the trial functions are themselves projected using $\mathbb{H}_{0}$ and the trial vectors $\phi_{1}=(1,0,0), \phi_{2}=(0,1,0), \phi_{3}=$ $(0,0,1)$ at the position $A_{0}$. These symmetry-adapted trial functions can then be used to perform the projection of the system's BVs.

This technique is particularly appropriate when the lowering of a systems symmetry divides related positions into a number of orbits. Consider some position $A_{i}=g_{i} A_{0}$, where $g_{i}$ consists of a rotation-reflection $h_{i}$ and a translation $\tau_{i}$. If $\mathbb{H}_{i}$ is the group of operations "stabilizing" $A_{i}$ then $\mathbb{H}_{i}=g_{i} \mathbb{M}_{0} g_{i}^{-1}$. Thus, if two orbits are related by the operation $g_{i}$, appropriate set of trial functions $\psi_{\text {trial }}$ are related by $h_{i} \psi_{\text {trial }}$.

$$
\begin{aligned}
\mathbb{H}_{i} & =g_{i} \mathbb{H}_{0} g_{i}^{-1}, \\
\psi_{\text {trial,orbit }_{i}} & =h_{i} \psi_{\text {trial,orbit }_{0}}
\end{aligned}
$$

In the next section we will work through an example where the standard trial functions produce an excess of solutions and determine a more appropriate trial set. The example splits into two orbits under the distorted ordering, and we show that the trial functions for both orbits have the relationship given in (9).

4.2. Worked Example. Consider the space group $I 4_{1} 32$ (214) and the $k$-vector $k=(1 / 2,1 / 2,1 / 2)$, with an atom at the position $(0,0,0)$. Under the operations of the space group of the $k$-vector, $G_{k}$, there are three equivalent positions at $(1 / 2,1 / 2,0),(0,1 / 2,1 / 2)$, and $(1 / 2,0,1 / 2)$. Using SARA $h$, the decomposition of possible atomic displacements is given as follows:

$$
\Gamma_{\mathrm{Mag}}=2 \Gamma^{1}+2 \Gamma^{2}+2 \Gamma^{3} .
$$

Every IR is of order 2, and therefore we expect $2 \times 2=4$ $\mathrm{BV}$ s to be projected from each IR.

The basis vectors generated for $\Gamma_{1}$ using the standard trial vectors $\phi_{1}=(1,0,0), \phi_{2}=(0,1,0), \phi_{3}=(0,0,1)$ are listed in Table 1, using the following notation:

$$
\psi_{i j}^{n}(x, y, z)=\left(\begin{array}{l}
a_{0} \\
b_{0} \\
c_{0}
\end{array}\right) A_{0}+\cdots
$$

BV $\psi_{i j}^{n}(x, y, z)$ has been projected from IR $\Gamma^{n}$, using the $i j$ th matrix element of each matrix and the trial vector $(x, y, z)$ at the position $A_{0}=(0,0,0)$. It consists of a series of vectors $\left(a_{n}, b_{n}, c_{n}\right)$, defined with respect to the crystallographic axes, at the positions $A_{n}$. Projection using the standard trial functions generates six apparently distinct BVs, rather than the four required by the reduction formula; clearly one of the trial functions is superfluous. It can be shown that $\psi_{11}^{1}(0,0,1)=e^{(2 / 3) \pi \cdot i} \psi_{11}^{1}(1,0,0)+e^{(-2 / 3) \pi \cdot i} \psi_{11}^{1}(0,1,0)$; however, this solution is neither apparent on inspection nor simple to determine.

Following the strategy of Section 4.2, we seek to determine a set of symmetry-adapted trial functions to simplify the $\mathrm{BV}$ relationships. The stabilizer group of the position $A_{0}$ is the group of $C_{3}$ rotations about $(1,1,1)$. The invariant subspaces of this group are the line $(1,1,1)$ and the perpendicular plane $[1,1,1]$. Thus we select one trial vector to lie along $(1,1,1)$ and the other two to lie in $[1,1,1]$ chosen to form right-hand sets $\phi_{1}=(1,1,1), \phi_{2}=(1,-1,0)$, and $\phi_{3}=(1,1,2)$. As presented in Table 2, these trial vectors have been renormalized to have modulus 1. Also listed are the BVs generated from the symmetry-selected set of trial functions. By inspection, $(1 / \sqrt{2}) \psi_{11}^{1}(1,-1,0)=-i \cdot(1 / \sqrt{6}) \psi_{11}^{1}(1,1,-2)$, and we can eliminate either $\phi_{2}=(1,-1,0)$ or $\phi_{3}=(1,1,-2)$ from our projection. Thus by suitable selection of trial functions we have produced a set of BVs in which excess solutions are readily discernible. 
TABle 1

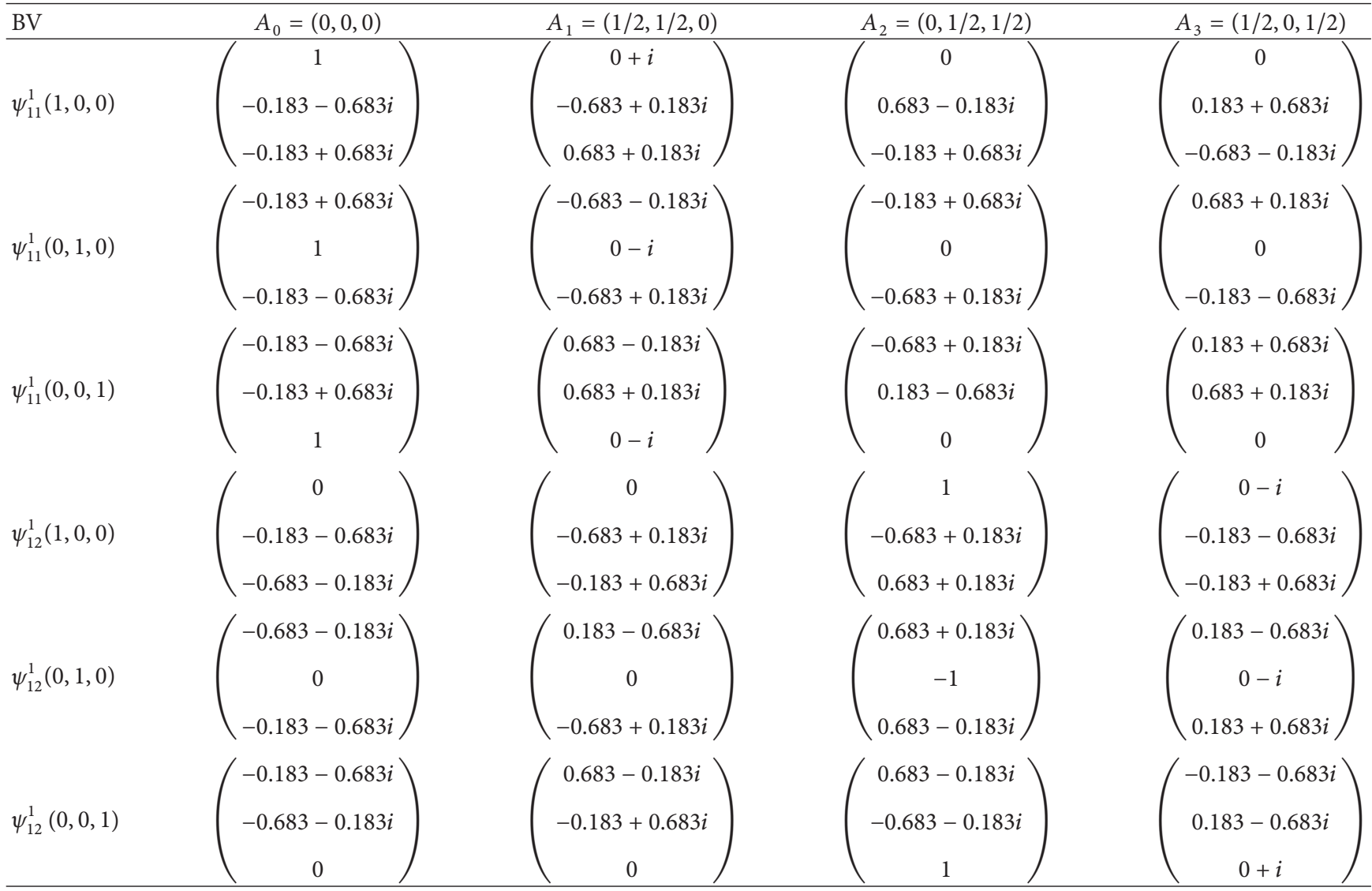

TABle 2

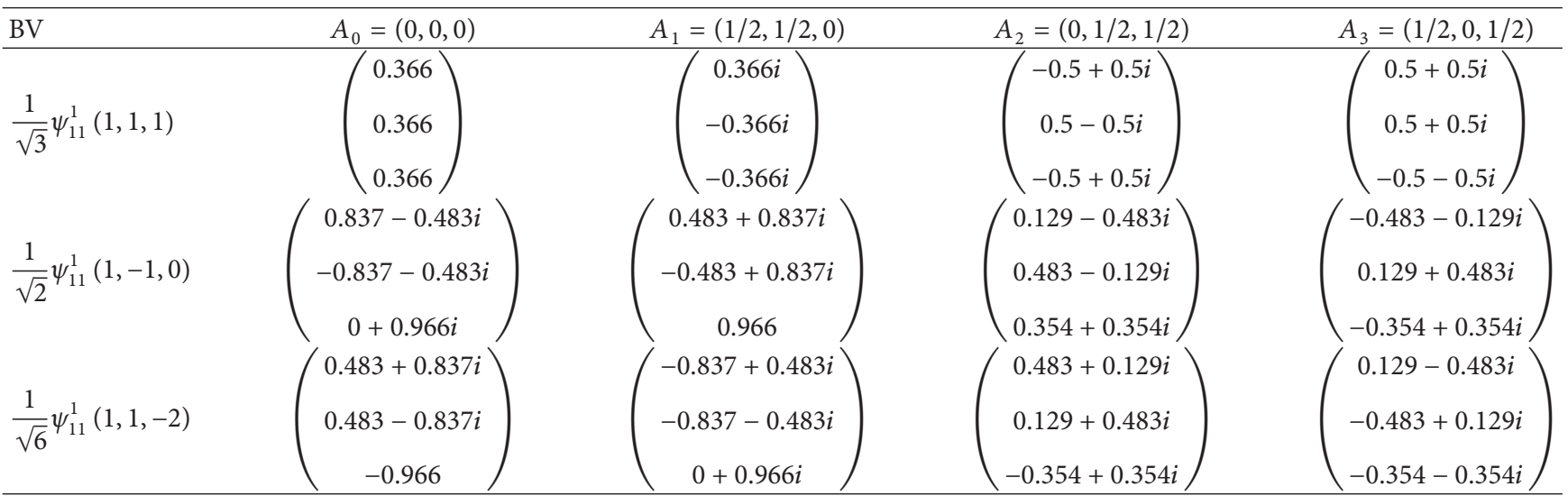

Further, our example is split into two orbits, the second orbit being related to the previously considered set of atomic positions by the following operation:

$$
h_{5}=\left(\begin{array}{ccc}
0 & 1 & 0 \\
1 & 0 & 0 \\
0 & 0 & -1
\end{array}\right) \text {. }
$$

Using (9), the following trial functions are generated for the second orbit: $\phi_{1}=(1,1,-1), \phi_{2}=(-1,1,0)$, and $\phi_{3}=(1,1,2)$ should generate BVs with the desired linear relationships. The BVs for the second orbit, using this set of trial functions, are presented in Table 3. Inspection reveals 
TABLE 3

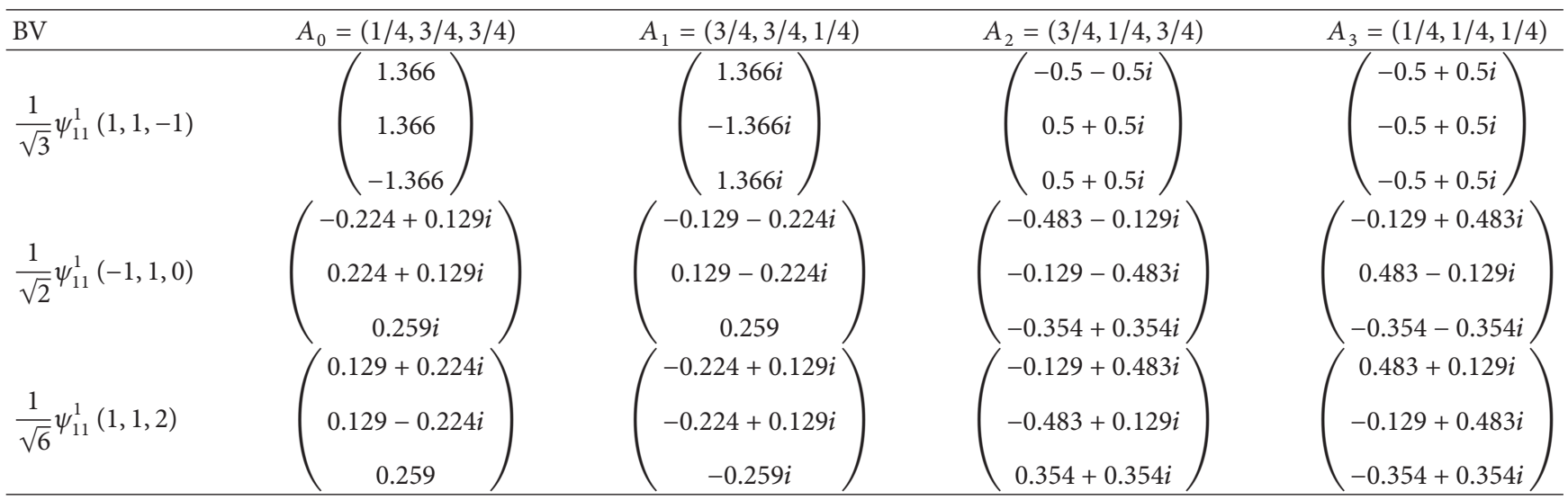

that $(1 / \sqrt{2}) \psi_{11}^{1}(-1,1,0)=i \cdot(1 / \sqrt{6}) \psi_{11}^{1}(1,1,2)$, and again we are free to eliminate either $\phi_{2}$ or $\phi_{3}$. We note that this method is also applicable to orbits joined under corepresentations.

\section{Undergeneration}

Undergeneration is the apparent inability to fully span a system's decomposition using the BVs generated by the method of projection operators. For the projection operator $W_{i j}^{\mu}$ changing $\mu$ generates a basis set with a different symmetry; hence the only free variable with which to resolve undergeneration is $i j$. This problem has been thoroughly explored by Stokes et al. [23], who define that when varying the column index $j$ will generate inequivalent BVs. It is useful to discuss this problem using basis sets to demonstrate the power of this concept in understanding the method of projection operators.

Basis vectors occur in basis sets which transform under two relations:

$$
\begin{aligned}
g \psi_{i}^{\mu} & =\sum_{j}^{d^{\mu}} \mathfrak{d}_{j i}^{\mu}(g) \psi_{j}^{\nu}, \\
W_{i j}^{\mu} \psi_{j}^{\mu} & =\psi_{i}^{\mu} .
\end{aligned}
$$

It is apparent from consideration of these two equations that the enumeration of BVs is not arbitrary; it defines how BVs interrelate within the basis set to which they belong. Further, the number of basis sets of a symmetry $\Gamma^{\mu}$ is exactly $C^{\mu}$, and within each set the BVs will be labelled $1,2, \ldots, d^{\nu}$. Thus, while the numbering is not arbitrary it is not unique either. The action of $W_{i 1}^{\mu}$ on a general vector $\psi$ is to project the component along $\psi_{1}^{\mu}$ into $\psi_{i}^{\mu}$. Similarly, the action of $W_{i 2}^{\mu}$ is to project the component along $\psi_{2}^{\mu}$ into $\psi_{i}^{\mu}$. However, there is no restriction that $\psi_{1}$ and $\psi_{2}$ are from the same basis set.

Thus, we conclude that varying the row-index $i$ generates another member of the same basis set, while varying the column index $j$ generates a BV from a different basis set (which may be equivalent).

\section{Unitary Check}

In Section 3 it was emphasised that the projection and reduction operators are normally encountered in a form which restricts the IRs to being unitary. Their use with nonunitary IRs would generate BVs lacking the correct symmetry properties. The absence of symmetry relations between the BVs could be realized as under- or overgeneration; therefore it is key to discuss possible sources of IRs for these calculations.

IRs derived from Zak's method [26] are, by derivation, unitary; the IRs outputs by the computer codes KAREP [27] and REPRES [28] are examples of such. However, when using IRs from collated tables the unitary properties must be confirmed explicitly. This was done for Kovalev's tables (1991) by Davies and Wills [29], and we reproduce an outline of the method here.

The method of verification was brute-force calculation. Our algorithm determined for each symmetry element $g_{1}$, some symmetry element $g_{2}$ for which $g_{1} g_{2}$ is an identitytranslation, represented by a complex number $C$. If the IR is a unitary homomorphism then $C^{*}$ will transform $\mathfrak{d}\left(g_{2}\right)$ into the conjugate transpose of $\mathfrak{d}\left(g_{1}\right)$ as follows:

$$
\begin{gathered}
\mathfrak{I}\left(g_{1}\right) \times \mathfrak{I}\left(g_{2}\right)=\left|\begin{array}{cccc}
1 & 0 & 0 & T_{x} \\
0 & 1 & 0 & T_{y} \\
0 & 0 & 1 & T_{z} \\
0 & 0 & 0 & 1
\end{array}\right|, \\
\mathfrak{d}^{\dagger}\left(g_{1}\right)=\mathfrak{d}\left(g_{2}\right) \times \exp \left(-2 \pi \times\left(k_{x}, k_{y}, k_{z}\right) \cdot\left(\begin{array}{c}
T_{x} \\
T_{y} \\
T_{z}
\end{array}\right)\right) .
\end{gathered}
$$

The matrices $\mathfrak{T}\left(g_{i}\right)$ are the normal $4 \times 4$ matrix representation of the affine operation $g_{i}$. The vector $\left(k_{x}, k_{y}, k_{z}\right)$, termed the $k$-vector, defines the translational periodicity of a modulated structure, and the representation of a translation is a function of its dot-product with the $k$-vector.

This work showed that the IRs presented in Kovalev's tables are indeed unitary and validate the projection and reduction techniques used in the computer codes based upon them, such as SARA $h[1]$. 


\section{Conclusions}

A common problem in the application of projection techniques to physical problems in crystallography is the overand undergeneration of basis vectors. Understanding the method in terms of basis sets allows a solution to overgeneration to be constructed through use, and subsequent elimination, of symmetry-adapted trial functions. This technique ensures that the solution is a set of basis sets and has all the required symmetry properties.

Further we show that the unitary nature of the IRs presented by Kovalev follows the restrictions placed upon the IRs by the projection operator and that any difficulties in the projection of a minimal spanning set of BVs are a consequence of this structure.

\section{Competing Interests}

The authors declare that they have no competing interests.

\section{Acknowledgments}

The authors would like to acknowledge the support of UCL in funding this work.

\section{References}

[1] A. S. Wills, "New protocol for the determination of magnetic structures using simulated annealing and representational analysis (SARAh)," Physica B: Condensed Matter, vol. 276-278, pp. 680-681, 2000.

[2] J. Rodríguez-Carvajal, "Recent advances in magnetic structure determination by neutron powder diffraction," Physica B: Physics of Condensed Matter, vol. 192, no. 1-2, pp. 55-69, 1993.

[3] A. Coelho, Topas Academic Version 5, Coelho Software, Brisbane, Australia, 2012.

[4] A. S. Wills, "Symmetry in the solid state; working beyond the space group," Journal of Materials Chemistry, vol. 15, no. 2, pp. 245-252, 2005.

[5] B. J. Campbell, J. S. O. Evans, F. Perselli, and H. T. Stokes, "IUCr computing," Commission Newsletter, vol. 8, pp. 81-95, 2007.

[6] A. S. Wills and Z. L. Davies, "Application of corepresentation theory to magnetic structures and displacive phase transitions," Acta Crystallographica A, vol. 64, p. C617, 2008.

[7] M. S. Senn, J. P. Wright, and J. P. Attfield, "Charge order and three-site distortions in the Verwey structure of magnetite," Nature, vol. 481, no. 7380, pp. 173-176, 2011.

[8] J. M. Perez-Mato, S. V. Gallego, L. Elcoro, E. Tasci, and M. I. Aroyo, "Symmetry conditions for type II multiferroicity in commensurate magnetic structures," Journal of Physics: Condensed Matter, vol. 28, no. 28, Article ID 286001, 2016.

[9] J. W. Lewis, J. L. Payne, I. R. Evans, H. T. Stokes, B. J. Campbell, and J. S. O. Evans, "An exhaustive symmetry approach to structure determination: phase transitions in $\mathrm{Bi}_{2} \mathrm{Sn}_{2} \mathrm{O}_{7}$," Journal of the American Chemical Society, vol. 138, no. 25, pp. 8031-8042, 2016.

[10] A. Poole, A. S. Wils, and E. Lelièvre-Berna, "Magnetic ordering in the $X Y$ pyrochlore antiferromagnet $\mathrm{Er}_{2} \mathrm{Ti}_{2} \mathrm{O}_{7}$ : a spherical neutron polarimetry study," Journal of Physics: Condensed Matter, vol. 19, no. 45, Article ID 452201, 2007.
[11] M. Boehm, B. Roessli, J. Schefer et al., "Complex magnetic ground state of $\mathrm{CuB}_{2} \mathrm{O}_{4}$," Physical Review B, vol. 68, no. 2, Article ID 024405, 2003.

[12] S. Calder, J. G. Vale, N. A. Bogdanov et al., "Spin-orbitdriven magnetic structure and excitation in the $5 \mathrm{~d}$ pyrochlore $\mathrm{Cd}_{2} \mathrm{Os}_{2} \mathrm{O}_{7}$," Nature Communications, vol. 7, article 11651, 2016.

[13] A. Lappas, A. S. Wills, M. A. Green, K. Prassides, and M. Kurmoo, "Magnetic ordering in the rutile molecular magnets $\mathrm{M}^{I I}\left[\mathrm{~N}(\mathrm{CN})_{2}\right]_{2}\left(M=\mathrm{Ni}, \mathrm{Co}, \mathrm{Fe}, \mathrm{Mn}, \mathrm{Ni}_{0.5} \mathrm{Co}_{0.5}\right.$, and $\left.\mathrm{Ni}_{0.5} \mathrm{Fe}_{0.5}\right)$, Physical Review B, vol. 67, no. 14, Article ID 144406, 2003.

[14] C. R. Wiebe, J. S. Gardner, S.-J. Kim et al., "Magnetic ordering in the spin-ice candidate $\mathrm{Ho}_{2} \mathrm{Ru}_{2} \mathrm{O}_{7}$," Physical Review Letters, vol. 93, no. 7, Article ID 076403, 2004.

[15] L. Tsujii, L. Keller, A. Dönni, and H. Kitazawa, "Anisotropic magnetic properties and magnetic structure of YbPdSi," Journal of Physics: Condensed Matter, vol. 28, no. 33, Article ID 336002, 2016.

[16] E. F. Bertaut, "Lattice theory of spin configuration," Journal of Applied Physics, vol. 33, no. 3, pp. 1138-1143, 1962.

[17] E. F. Bertaut, "On group theoretical techniques in magnetic structure analysis," Journal of Magnetism and Magnetic Materials, vol. 24, no. 3, pp. 267-278, 1981.

[18] C. J. Bradley and A. P. Cracknell, Mathematical Theory of Symmetry in Solids, Clarendon Press, Oxford, UK, 1972.

[19] Y. A. Izyumov and V. E. Naish, Neutron Diffraction of Magnetic Materials, Consultants Bureau, New York, NY, USA, 1991.

[20] W. Sikora, F. Białas, and L. Pytlik, "MODY: a program for calculation of symmetry-adapted functions for ordered structures in crystals," Journal of Applied Crystallography, vol. 37, no. 6, pp. 1015-1019, 2004.

[21] W. Sikora and L. Pytlik, "Application of symmetry analysis to description of ordered structures in crystals," in Encyclopedia of Mathematics Research, J. D. Mathias and S. L. Cleaves, Eds., Nova Science Publishers, New York, NY, USA, 2012.

[22] Y. A. Izyumov and Syromyatnikov, Phase Transitions and Crystal Symmetry, V. N. Kluwer Academic, London, UK, 1990.

[23] H. T. Stokes, D. M. Hatch, and J. D. Wells, "Group-theoretical methods for obtaining distortions in crystals: applications to vibrational modes and phase transitions," Physical Review B, vol. 43, no. 13, pp. 11010-11018, 1991.

[24] O. V. Kovalev, Representations of the Crystallographic Space Groups: Irreducible Representations, Induced Representations and Corepresentations, Gordon and Breach Science, Amsterdam, The Netherlands, 1993.

[25] M. Hamermesh, Group Theory and Its Application to Physical Problems, Addison-Wesley, Reading, Pa, USA, 1964.

[26] J. Zak, "Method to obtain the character tables of nonsymmorphic space groups," Journal of Mathematical Physics, vol. 1, no. 3, p. $165,1960$.

[27] E. Hovestreydt, M. Aroyo, S. Sattler, and H. Wondratschek, "KAREP - a program for calculating irreducible space-group representations," Journal of Applied Crystallography, vol. 25, no. 4, p. 544, 1992.

[28] E. Kroumova, M. I. Aroyo, J. M. Perez-Mato et al., "Bilbao crystallographic server: useful databases and tools for phasetransition studies," Phase Transitions: A Multinational Journal, vol. 76, no. 1-2, pp. 155-170, 2003.

[29] Z. L. Davies and A. S. Wills, "A new verification of Kovalev's tables of irreducible representations of the space groups," Journal of Physics: Condensed Matter, vol. 20, no. 10, Article ID 104232, 2008. 

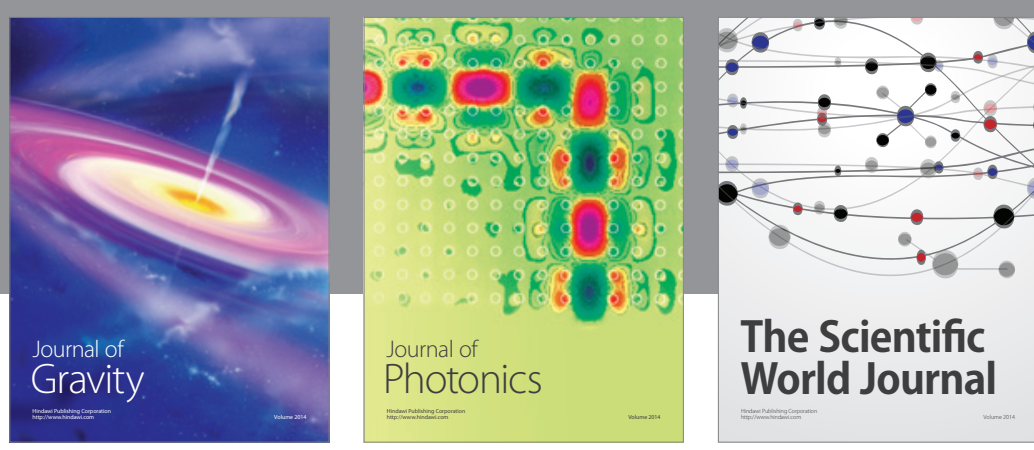

The Scientific World Journal
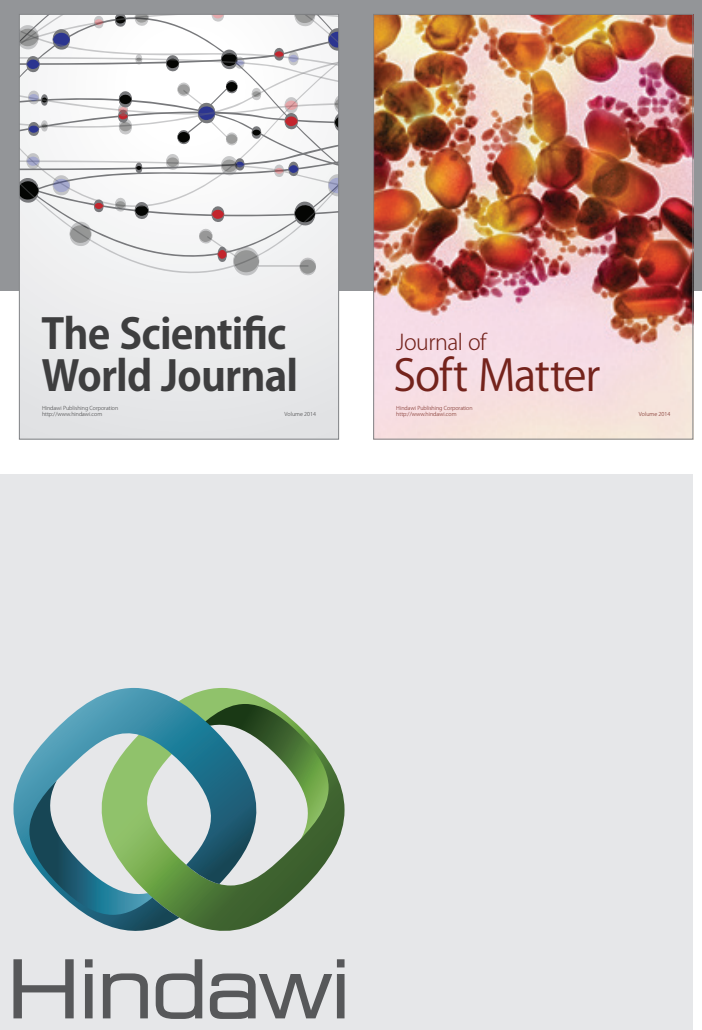

Submit your manuscripts at

http://www.hindawi.com

nternational Journal of

Statistical Mechanics
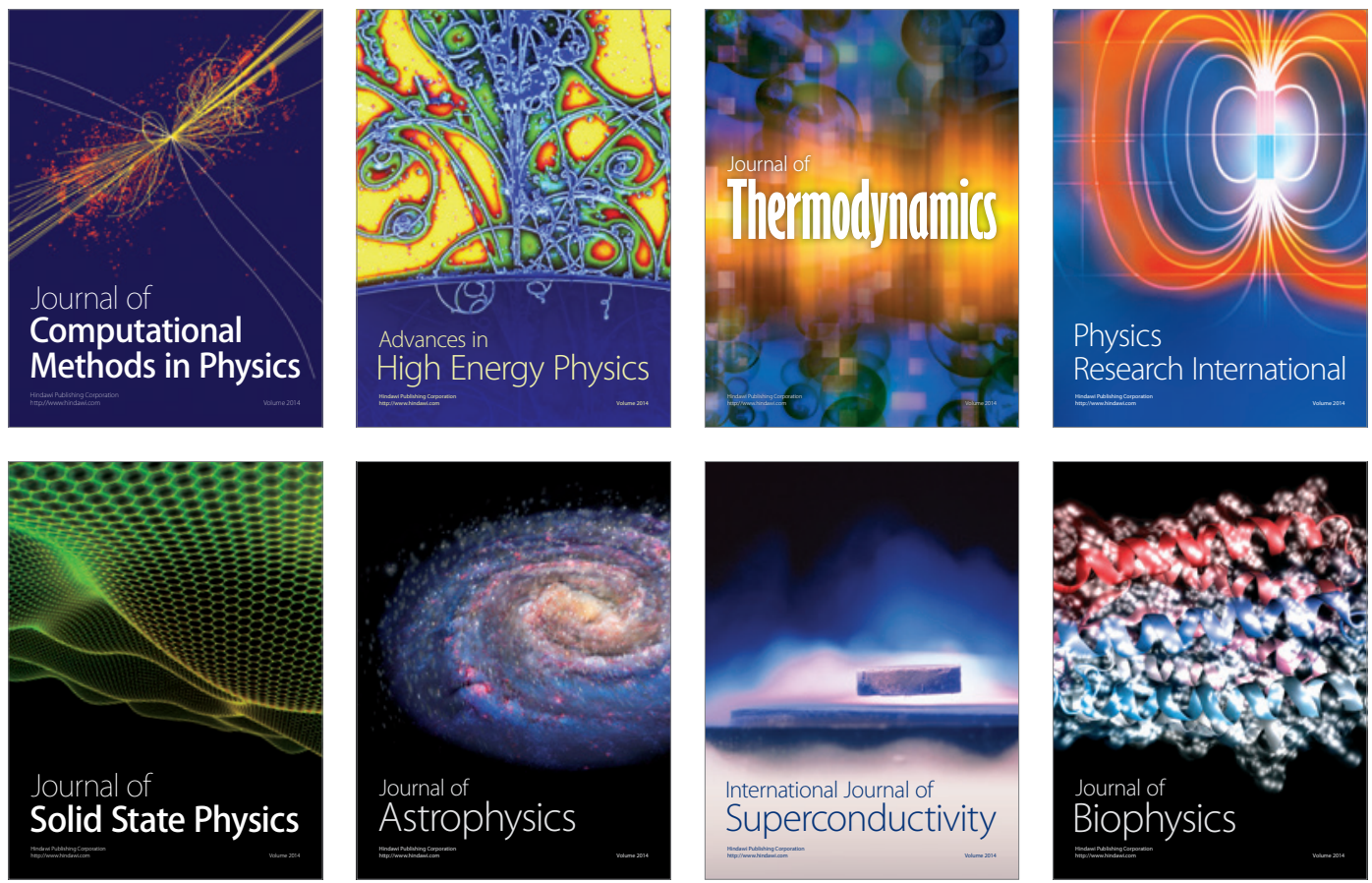
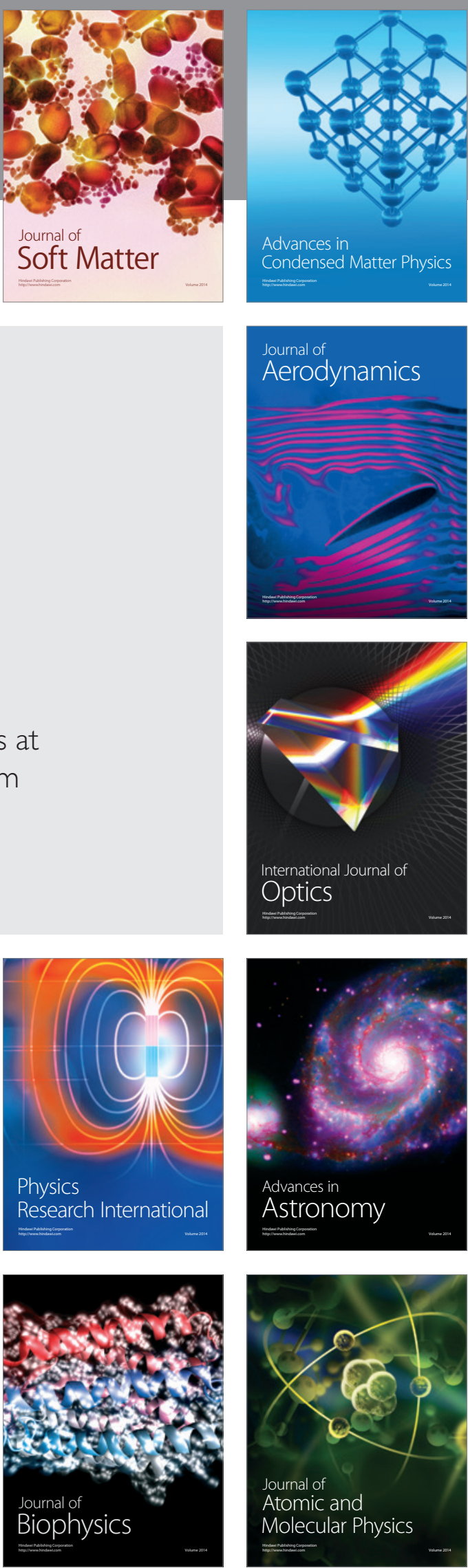